\title{
Interactive comment on "Assessment of shallow landslide susceptibility using an artificial neural network in Enshi region, China" by Bin Zeng et al.
}

\section{Bin Zeng et al.}

zengbin_19@126.com

Received and published: 25 August 2017

\section{Dear Referee and Editor:}

Thank you for the valuable suggestions. We have carefully read through the comments, and our responses to the referee's questions are listed below. We greatly appreciate your time and efforts to improve our manuscript for further revision and publication.

1. The manuscript has very limited contribution to the field since many papers have been published in the last two decades on the use of neural nets in landslide susceptibility mapping. Authors should explain how they choose the parameters (learning rate, momentum, initial weight range) and why? For example when you choose 10.000 iterations in training stage, the network is high likely to overfit the data that is extremely

Printer-friendly version

Discussion paper 
limited in size. Re: Compare with existing studies, some new attempts have been carried out in this paper: (1) this research focused on the distribution of unstable slope zones rather than the existing landslides, since the unstable slopes are more dangerous; (2) this research predicted the unstable slope distribution only in Silurian stratum so as to avoid the interference due to differences in slope (in different stratum) failure mechanisms; (3) a "slope structure thematic map" was taken into account to better represent the especial slope failure mechanism in Silurian stratum; (4) replaced the temporal variable of rainfall into a static, spatial variable termed "catchment area" to better act as an influencing factor during the landslide susceptibility. The research results can provide useful guidance for both landslide susceptibility assessment and land planning processes. In the chapter 3.2.2, the paper explained the decision process of many parameters like "the number of neurons for the input and output layers", "the number of hidden layers", "the number of neurons in the hidden layer", "the network training function". And In the BP neural network, "the initial network weights and thresholds" were given random values in the acceptable range, based on the theory that an initial value which is not too large has little impact on the overall performance of the network, while a smaller initial range is more conducive to uniformly random initial weights (Freeman, 1993). The author has carried out a lot of tentative calculations, so as to compare the ability of different training functions (traingd, traingdm, traingdx, trainlm etc.). After choosing "traingdx" as the training function of the neural network, the author also carried out a lot of tentative calculations to determine the number of training iteration (10000 times), so as to ensure that the network can achieve the training goal and converge.

2. Another important problem is related to the sample size, which I believe is too limited, for the designed network (4-9-1). This network has totally 45 links. The number of training samples employed at the learning stage has a significant impact on the performance of any classifier. This issue is perhaps more important for neural networks than for conventional statistical classifiers since their performance is totally dependent upon the characteristics of the training data presented. Although the size of the train-

Printer-friendly version Discussion paper 
ing data is of considerable importance, the characteristics and the distributions of the data as well as the sampling strategy used are crucial. The quality and the quantity of the training samples are crucially important for a successful neural network application. Whilst too few training samples are not sufficient for neural networks to derive the characteristics of the classes, the use of too large a number of training samples may cause networks to overfit to the data, as well as requiring more time for learning. Re: The final forecasting area of this research is about $103 \mathrm{~km} 2$, which is the range of Silurian stratum in Enshi region. In this area, 35 stable and unstable slopes in Silurian stratum in Enshi region were chosen as the sample data, the recognition and mapping work have been carried out by geomorphological field survey. All the 35 chosen samples fitted the failure mechanism of landslides in Silurian stratum as discussed in chapter 2.2.2 and 2.2.3 and covered as much as possible the different combinations of various factors to improve the forecasting ability of the network. So that the neural network trained by these samples, can effectively approximate the inherent law of the samples by studying and remembering the known samples, then carry out an associated forecast according to the memory.

3. On page 4 lines 54 and 61 the surname of the author (Pourghasemi) was written incorrectly. On page 6 line 87 "compare with... some different attempts" can be replaced as "compared with .... some attempts". Re: The author will correct this mistake, and also check the whole manuscript to avoid similar mistakes.

4. Conclusion section is too short and includes general information. Re: The author will further revise the conclusion chapter: adding more discussion about the research significance and novelty, and simplify the general information.

5. Provide more recent papers in reference list in the use of ANNs and its performance comparison to machine learning methods. Re: The author will supply more recent references concerning the use of ANNs and its performance comparison to machine learning methods, and will also discuss these research results in the proper chapter of the paper. 
Interactive comment on Nat. Hazards Earth Syst. Sci. Discuss., https://doi.org/10.5194/nhess2017-176, 2017.
NHESSD

Interactive

comment 